ME podría resentirse y, asimismo, supondría una dificultad para el acuerdo de reconocimiento mutuo con la Asociación Médica Americana (AMA), renovado en julio de 2006 por un período de cuatro años.

Desde el punto de vista de los organizadores-proveedores de actividades de FMC, el valor añadido radica en la dimensión internacional que permite. De lo que deriva que un mayor número de potenciales participantes extranjeros puedan estar interesados en la actividad en cuestión.

Los beneficios a largo plazo derivan de la interrelación entre los organismos acreditadores nacionales. Dichos organismos están vivamente interesados en mantener sus prerrogativas a nivel nacional y adjudicar los créditos a los médicos del país. EACCME representa una institución en la que ellos participan activamente, con su autoridad correspondiente, de lo que se deriva una facilitación del intercambio de los créditos, de manera similar a como se reconocen mutuamente los diplomas de postgrado. De hecho, los organismos acreditadores nacionales trabajan con los organismos reguladores de la profesión para la concesión de la licencia de ejercicio

Un último objetivo es el desarrollo de un sistema que haga más fácil la vida profesional de nuestros colegas, ofreciéndoles un mecanismo de reconocimiento universal de los créditos de FMC que les garantice su utilización para los requerimientos que se establezcan.

\section{Retos de la formación médica continuada. Situación actual y perspectivas de futuro en España}

Luis Pallarés' y Hélios Pardell².

1. Secretario Técnico de la Comisión de Formación Continuada del Sistema Nacional de Salud. Madrid, España.

2. Director del Sistema Español de Acreditación de la Formación Médica Continuada (SEAFORMEC). Madrid, España

A la vista de la situación de la formación médica continuada (FMC) y, concretamente, de su acreditación en el contexto europeo, pormenorizadamente analizada por el Dr. B. Maillet en la ponencia que abre esta sesión, no podemos por menos que sentirnos satisfechos de los desarrollos llevados a cabo en España en los últimos 10 años, que nos han coloca- do en una posición inmejorable para hacer frente a los retos de futuro.

Pero no por ello debemos quedarnos anclados en las estructuras diseñadas y los procesos implementados. Por el contrario, debemos proseguir en el camino de mejorar constantemente la oferta formativa, por un lado, y el sistema de acreditación de la misma, por el otro.

Por ello, aquí vamos a describir someramente la situación actual de la acreditación de la FMC desde la perspectiva de la Comisión de Formación Continuada del SNS y las Comisiones Autonómicas, de un lado, y del Sistema Español de Acreditación de la FMC (SEAFORMEC), de otro.

$\mathrm{Y}$, en función de lo que decíamos más arriba, vamos a ofrecer algunas pinceladas sobre las líneas de futuro que, a nuestro juicio, deberían guiar los pasos del sistema español de acreditación en su progresión a medio y largo plazo. 


\section{ANTECEDENTES}

Por la década de los 90 s diversas sociedades científicas implantaron sus sistemas propios de acreditación de la FMC, alguno de los cuales, como el SAAP todavía tiene vigencia parcial. Asimismo, la Organización Médica Colegial creaba su Instituto de Formación Médica, diseñado de acuerdo con las tendencias internacionales más modernas, que apenas llegó a iniciar su actuación, desapareciendo a poco más de un año de su creación. Y la Consejería de Sanidad de la Comunidad Autónoma de Madrid, introduciría su sistema preliminar de reconocimiento-acreditación de actividades formativas.

Pero el primer sistema comprensivo de acreditación, básicamente inspirado en el sistema norteamericano, fue el del Consejo Catalán de la Formación Médica Continuada, creado en 1989 y que iniciaría su actividad acreditadora para el conjunto de la profesión médica catalana en 1997. A partir de sus presupuestos metodológicos, se desarrollaría el sistema de acreditación de la Comisión de Formación Continuada del Sistema Nacional de Salud, que supuso el arranque del movimiento español de acreditación.

\section{LA COMISIÓN DE FORMACIÓN}

CONTINUADA DEL SISTEMA NACIONAL DE SALUD Y LAS COMISIONES AUTONÓMICAS

En diciembre de 1997 los Ministerios de Sanidad y Consumo y de Educación, Cultura y Deporte firmaban con las 17 Comunidades Autónomas el Convenio de Conferencia Sectorial del Consejo Interterritorial del Sistema Nacional de Salud por el que se procedía a la creación de la Comisión de Formación Continuada del SNS.

En dicho Convenio se preveía el desplegamiento a nivel autonómico del sistema, con la creación de las correspondientes Comisiones Autonómicas de Formación Continuada, a imagen y semejanza de la Comisión antes mencionada.

Los desarrollos legislativos posteriores, básicamente la Ley de Ordenación de las Profesiones Sanitarias (LOPS), de 21 de Noviembre de 2003, contemplan la regulación del marco de la FMC, a partir de la cual se prevé la obtención de titulaciones especiales diferenciadas de las otras dos titulaciones fundamentales: la de especialidad y la de capacitación específica. Dichas titulaciones especiales son los denominados Diplomas de Acreditación y de Acreditación Avanzada.
El último desarrollo legislativo, a partir de las disposiciones de la Ley de Cohesión y Calidad del Sistema Nacional de Salud, de 28 de Mayo de 2003, y de la LOPS, ha sido el proyecto de Real Decreto por el que se determina la composición y funciones de la Comisión de Formación Continuada de las Profesiones Sanitarias, pendiente de su aprobación definitiva cuando redactamos este artículo (septiembre de 2006).

A lo largo de sus casi diez años de funcionamiento efectivo, el sistema de acreditación de la Comisión ha demostrado no solo su factibilidad sino, además, su impacto sobre la mejora de la oferta formativa. Por otro lado, las previsiones de desplegamiento autonómico se han visto cumplidas en su práctica totalidad dado que, a fecha de hoy (septiembre de 2006), quince de las diecisiete Comunidades Autónomas disponen de su propia Comisión de Formación Continuada, por más que sólo son operativas 10 de ellas.

Desde su creación, el sistema de la Comisión y de las Comisiones Autonómicas acredita actividades formativas para todas las profesiones sanitarias, aceptando tanto actividades presenciales como a distancia o mixtas. En función de su trayectoria histórica, en Cataluña existen los Consejos Catalanes de Formación Médica Continuada y de Formación Farmacéutica Continuada, que acreditan actividades de cada uno de los colectivos profesionales específicos, habiéndose creado recientemente el Consejo Catalán de Formación Continuada de las Profesiones Sanitarias, que engloba a los dos anteriores y prevee la creación de otros Consejos Técnicos específicos para otros colectivos profesionales sanitarios. El Consejo Catalán de Formación Continuada de las Profesiones Sanitarias puede equipararse, de hecho, a las Comisiones Autonómicas de Formación Continuada existentes a nivel del resto de Comunidades Autónomas y, junto a ellas, se halla representado en la Comisión Nacional de Formación Continuada del SNS.

La metodología de acreditación, en manos de las secretarías técnicas correspondientes, es estrictamente equivalente y los créditos adjudicados a las actividades acreditadas son de validez universal en todo el territorio nacional.

\section{EL SISTEMA ESPAÑOL DE ACREDITACIÓN DE LA FMC (SEAFORMEC)}

En fecha 8 de Febrero de 2002, los Ministerios de Sanidad y Consumo y de Educación, Cultura y Deporte firmaban con los Consejos Generales de 
Colegios Oficiales de Médicos, Farmacéuticos, Veterinarios, Odontoestomatólogos y Enfermeras sendos convenios de colaboración en materia de formación continuada, por los que se les encomendaba, entre otras funciones, la acreditación de las actividades de formación continuada de sus sectores profesionales específicos, bajo la tutela y supervisión de la Comisión de Formación Continuada del SNS.

A partir de dicho convenio, el 29 de Enero de 2003, el Consejo General de Colegios Oficiales de Médicos firmaba con la Federación de Asociaciones Científico-Médicas de España (FACME), la Conferencia de Decanos de Facultades de Medicina y el Consejo Nacional de Especialidades Médicas el acuerdo de creación del Sistema Español de Acreditación de la Formación Médica Continuada (SEAFORMEC). Este mismo año se constituía el sistema de acreditación para enfermería promovido por el Consejo General de Colegios de Enfermeras.

SEAFORMEC iniciaba su actividad de acreditación en junio de 2003 y, desde entonces, ha ido consolidando su posición como sistema acreditador de referencia para el sector médico español, diseñando líneas estratégicas de futuro tendentes a involucrar más activamente los diversos colectivos profesionales médicos, a imagen y semejanza de lo que ocurre en los países de nuestro entorno y a nivel internacional en general.

La metodología de acreditación utilizada por SEAFORMEC es estrictamente equivalente a la del sistema de la Comisión y, lógicamente, los créditos adjudicados tienen idéntica validez a todos los niveles. Adicionalmente, SEAFORMEC firmó un convenio de reconocimiento mutuo de los créditos con UEMS-EACCME, lo que significa que el curriculum de FMC de los médicos españoles es reconocido por los países integrados en UEMS-EACCME, en el contexto de la libre circulación de profesionales en el seno de la Unión Europea.

\section{SITUACIÓN ACTUAL Y LÍNEAS DE FUTURO}

Así pues, en la actualidad, en España, existen dos sistemas de acreditación de actividades de FMC para los médicos: el de la ComisiónComisiones Autonómicas y el de SEAFORMEC. En función de la utilización de la misma metodología de acreditación y de las previsiones legislativas, los créditos adjudicados por cada uno de ellos son equivalentes y tienen idéntico valor de uso para los fines propios (carrera profesional, desarrollo profesional continuo y otros sistemas de promoción profesional). Dado el carácter voluntario de la acreditación, se deja en manos de los proveedores-organizadores de las actividades formativas la elección del sistema de acreditación preferido.

Esta situación permite abrigar esperanzas fundadas de desarrollo y adecuación a las necesidades cambiantes de cada momento. Por otro lado, hoy por hoy, el sistema español ocupa un lugar destacado en el contexto europeo, en el que se perfila como un sistema de referencia que ha sabido resolver algunos de los problemas mayores relacionados con la FMC y su acreditación.

De ello no cabe deducir, sin embargo, que deba permanecer estático en el tiempo sinó que, por el contrario, debe saber hacer frente a los retos más importantes de futuro inmediato.

Entre tales retos descuellan, a nuestro juicio, los tres siguientes: los desarrollos del propio sistema de acreditación, la utilización adecuada de los créditos y el deplegamiento de las previsiones legislativas relacionadas con la FMC.

Por lo que se refiere al desarrollo del propio sistema de acreditación, en el que activamente están trabajando las secretarías técnicas en los últimos tiempos, hay que resolver los problemas planteados por la acreditación de las actividades formativas a distancia (no estará de más recordar que, justamente por tales problemas, algunos sistemas de acreditación de otros países no acreditan las actividades no presenciales); la equivalencia de los créditos españoles con los de otros países (SEAFORMEC ha hecho un intento previo en este sentido al incluir las horas lectivas equivalentes a los créditos adjudicados); la definición de requerimientos más estrictos para la acreditación; el despliegue de una normativa de esponsorización comercial-conflicto de intereses más comprensiva y transparente; y, por último, la aceptación de la acreditación institucional (introducida en Cataluña desde el inicio y contemplada por algunas Comunidades Autónomas en la actualidad).

En cuanto a la utilización de los créditos hay que, por un lado, normalizar su aplicación a efectos de la carrera profesional y del desarrollo profesional continuo a nivel estatal y, por otro, contemplar su aplicación a iniciativas de revalidación profesional (relicencia-recolegiación o recertificación) que ineludiblemente deben llevar a cabo las asociaciones profesio- 
nales, en colaboración con la administración, con objeto de desarrollar las previsiones de la LOPS.

En relación con esta última, hay que recordar que claramente establece el acceso a los Diplomas de Acreditación y de Acreditación Avanzada a partir de la FMC acreditada, como vías complementarias de obtención de algún tipo de titulación, independiente de los de la especialidad y del área de capacitación específica. Ni que decir tiene que el desplegamiento de tales aspectos contemplados en la LOPS actualmente resulta prioritarios, como lo es el de las potencialidades del desarrollo profesional continuo (DPC), como alternativa a la carrera profesional (CP), adecuadamente contempladas en otra sesión.

\section{Bibliografía}

1. Barón M. Experiencias preliminares de acreditación de la formación médica continuada en España. El Instituto de Formación Médica de la Organización Médica Colegial. JANO. 1999; 57: 52-4

2. Castejón M. Sistemas de acreditación de formación continuada de profesiones sanitarias en España. Med Clin (Barc). 2004; 123: 224-9

3. Gual A, Pardell H. El Consejo Catalán de la Formación Médica Continuada. JANO. 1999; 57: 60-1

4. Gual A, Palés J, Pardell H, Oriol-Bosch A. Doctors in Spain. Clin Teacher. 2005; 2: 59-63

5. Oriol-Bosch A, Pardell H, directores. La profesión médica. Retos del milenio. Barcelona: Fundación Medicina y Humanidades Médicas; 2004

6. Pallarés L. El sistema de acreditación de la Comisión de Formación Continuada del Sistema Nacional de Salud y de las Comisiones Autonómicas. En: Pardell H, director. Industria farmaceútica y formación médica continuada. Barcelona: Ediciones MAYO, SA; 2003. 47-61

7. Pallarés L, Segura H. La acreditación de la formación médica continuada a distancia en los sistemas de la Comisión de Formación Continuada del Sistema Nacional de Salud y del Consejo Catalán de la Formación Médica Continuada. En: Pardell H, director. Formación médica continuada a distancia. Barcelona: Ediciones MAYO, SA; 2004. 63-74

8. Pallarés L. La acreditación de la formación médica continuada en el Sistema Nacional de Salud. Boletín de FMC/DPC. 2006 (en prensa)

9. Pardell H, Pallarés L. La situación actual de la formación médica continuada. Dónde estamo y hacia dónde vamos. JANO. 2001; 60: 31-3

10. Pardell H, Pallarés L. El futuro del sistema de acreditación en España. En: Pardell H, director. Formación médica continuada, a debate. Barcelona: Ediciones MAYO, SA; 2001. 113-8

11. Pardell H, Bruguera M. ¿ Formación médica continuada o desarrollo profesional continuo del médico?. Educación Médica. 2002; 5: 90-5

12.Pardell H, Oriol-Bosch A, en nombre de la Comisión Permanente del Consejo Catalán de la Formación Médica Continuada. Sobre la acreditación de la formación médica continuada. Med Clin (Barc). 2003; 120: 261-4

13. Pardell H, Sierra G. Continuing medical education and continuing medical education accreditation in Spain. JCEHP. 2003; 23: 244-6

14. Pardell H, Ramos A, Aliaga J, Cabero L, Contreras E, Gual A et al. Utilización de los créditos de formación médica continuada para la recertificación-relicencia profesional. Descripción de la primera experiencia española de recertificación-relicencia colegial voluntaria. Educación Médica. 2003; 6: 127-33

15.Pardell H. El Sistema Español de Acreditación de la Formación Médica Continuada (SEAFORMEC). JANO. 2003; 65: 50

16. Pardell H, Gual A, Pallarés L. Proceso de acreditación de la formación médica continuada en España. Barcelona: Ediciones MAYO, SA: 2003

17. Pardell H. De la acreditación de la formación médica continuada a la Ley de Ordenación de las Profesiones Sanitarias. Sobre la regulación de la profesión médica en España. Med Clin (Barc). 2004; 122: 267-8

18. Pardell $\mathrm{H}$, director. Industria farmaceútica y formación médica continuada. Barcelona: Ediciones MAYO, SA; 2004

19. Pardell H, director. Formación médica continuada a distancia. Barcelona: Ediciones MAYO, SA; 2004

20. Pardell H. Hacia la homologación internacional de los créditos de formación médica continuada. Declaración de un Comité de Expertos. Med Clin (Barc). 2005; 125: 14-5

21. Pardell H, Gual A, Segura H. Acreditación de la formación continuada de las profesiones sanitarias en España. Perspectivas futuras. Med Clin (Barc). 2006; 126: 380-3

22. Pardell H, Gómez Asorey C. ¿ De quién es responsabilidad la formación continuada de los médicos ?. Atenc Primaria. 2006; 37: 350-4

23. Pardell H. Esponsorización comercial de la formación médica continuada y conflicto de intereses. Med Clin (Barc). 2006; 127: 222-6

24. Pardell H, Pallarés L, Segura H. De la formación médica continuada al desarrollo profesional continuo. JANO, 2006 (en prensa)

25. Prieto A. Experiencias preliminares de acreditación de la formación médica continuada en España. Acreditación de actividades de formacióin continuada en atención primaria. JANO. 1999; 57: 48-9

26. Ramírez J, Pallarés L, Pardell H. El sistema español de acreditación de la formación médica continuada. Características generales. JANO. 1999; 57: 62-4

27. Rodríguez R. Experiencias preliminares de acreditación de la formación médica continuada en España. Sociedades científicas. JANO. 1999; 57: 50-1 